\title{
Comprehensive Analysis of lncRNAs Related to the Prognosis of Esophageal Cancer Based on ceRNA Network and Cox Regression Model
}

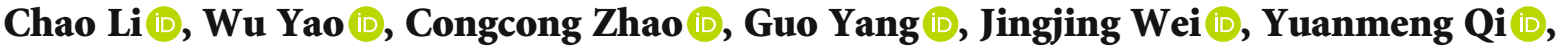 \\ Ruoxuan Huang $\mathbb{D}$, Qiuyan Zhao $\mathbb{D}$, and Changfu Hao $\mathbb{D}$
}

College of Public Health, Zhengzhou University, 450000, China

Correspondence should be addressed to Changfu Hao; haochangfu@126.com

Received 14 August 2020; Revised 15 November 2020; Accepted 21 November 2020; Published 11 December 2020

Academic Editor: Hassan Dariushnejad

Copyright (C) 2020 Chao Li et al. This is an open access article distributed under the Creative Commons Attribution License, which permits unrestricted use, distribution, and reproduction in any medium, provided the original work is properly cited.

\begin{abstract}
Background. Esophageal cancer is one of the most deadly malignant tumors. Among the common malignant tumors in the world, esophageal cancer is ranked seventh, which has a high mortality rate. Long noncoding RNAs (lncRNAs) play an important role in the occurrence and development of various tumors. IncRNAs can competitively bind microRNAs (miRNAs) with mRNA, which can regulate the expression level of the encoded gene at the posttranscriptional level. This regulatory mechanism is called the competitive endogenous RNA (ceRNA) hypothesis, and ceRNA has important research value in tumor-related research. However, the regulation of lncRNAs is less studied in the study of esophageal cancer. Methods. The Cancer Genome Atlas (TCGA) database was used to download transcriptome profiling data of esophageal cancer. Gene expression quantification data contains 160 cancer samples and 11 normal samples. These data were used to identify differentially expressed lncRNAs and mRNAs. miRNA expression data includes 185 cancer samples and 13 normal samples. The differentially expressed RNAs were identified using the edgeR package in $\mathrm{R}$ software. Then, the miRcode database was used to predict miRNAs that bind to lncRNAs. MiRTarBase, miRDB, and TargetScan databases were used to predict the target genes of miRNAs. Cytoscape software was used to draw ceRNA network. Gene Ontology (GO) and Kyoto Encyclopedia of Genes and Genomes (KEGG) analyses were performed using DAVID 6.8. Finally, multifactor cox regression was used to screen lncRNAs related to prognosis. Results. We have screened 1331 DElncRNAs, 3193 DEmRNAs, and 162 DEmiRNAs. Among them, the ceRNA network contains 111 lncRNAs, 11 miRNAs, and 63 DEmRNAs. Finally, we established a prediction model containing three lncRNAs through multifactor Cox regression analysis. Conclusions. Our research screened out three independent prognostic lncRNAs from the ceRNA network and constructed a risk assessment model. This is helpful to understand the regulatory role of lncRNAs in esophageal cancer.
\end{abstract}

\section{Introduction}

Esophageal cancer (EC) is one of the most deadly malignant tumors. Among the common malignant tumors in the world, EC is ranked seventh, which has a high mortality rate [1]. There are two main subtypes of esophageal cancer: esophageal squamous cell carcinoma (ESCC) is mainly distributed in Asia, Africa, and South America; esophageal adenocarcinoma (EAC) is mainly distributed in North America and Europe [2, 3]. Nowadays, surgical resection can improve the quality of life of patients with EC and prolong the survival time of patients, but the risk of surgical treatment is high. In addition, the use of surgical aids such as chemotherapy or chemoradiation to treat esophageal cancer has improved the prognosis of patients with advanced cancer, but the five-year survival rate of esophageal cancer is only $15 \%$ $25 \%$ [4-6]. Therefore, there is an urgent need to find molecular biomarkers for EC, which can help improve the prognosis and treatment of patients with EC.

Long noncoding RNA (lncRNA) is defined as RNA transcripts with more than $200 \mathrm{nt}$ and no coding ability [7]. There is increasing evidence that lncRNAs can regulate tumor genesis, including tumor cell proliferation, metastasis, differentiation, apoptosis, and metabolism [8-10]. Among them, 


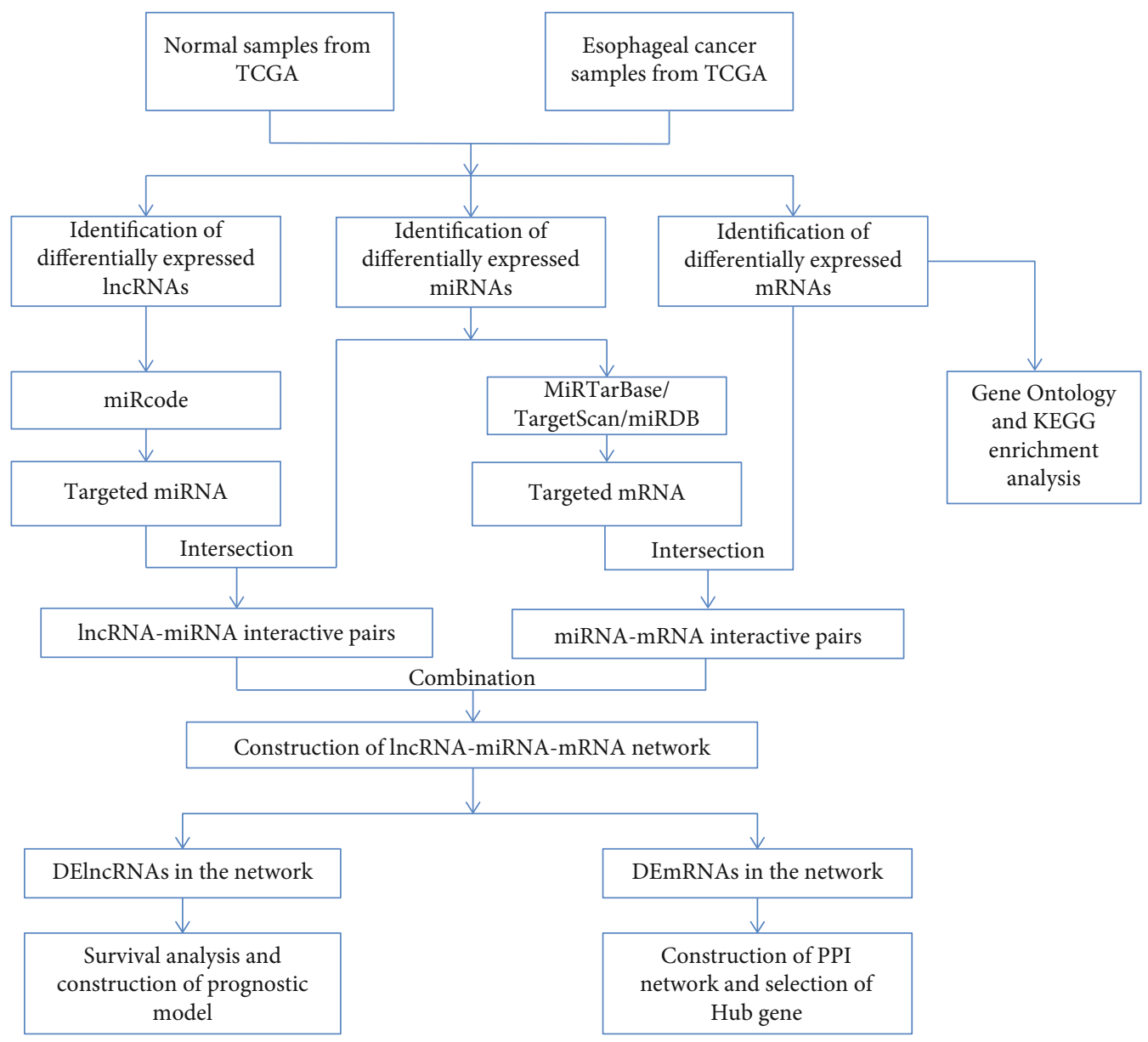

FIGURE 1: ceRNA network construction flowchart.

MALAT1, AFAP1-AS1, HOTAIR, TUG1, and MEG3 have been shown to be dysregulated in EC and they can regulate the occurrence and development of EC [11-16]. In addition, related studies have shown that lncRNAs may become prognostic markers for EC [17].

lncRNAs can regulate tumor genesis in many ways. When located in the nucleus, they are mainly involved in the process of transcription and epigenetics. When located in the cytoplasm, they participate in posttranscriptional regulation mainly by forming specific protein complexes or as ceRNA [18]. In 2011, Salmena et al. proposed the hypothesis of competitive endogenous RNA [19]. With the deepening of research related to lncRNA, the research of competitive endogenous RNA hypothesis is also increasing, which has become a hotspot in disease research.

In our study, the expression data of lncRNAs, miRNAs, and mRNAs related to EC samples and normal samples came from TCGA database. The differentially expressed RNAs were selected. Subsequently, Gene Ontology (GO) and Kyoto Encyclopedia of Genes and Genomes (KEGG) analyses were used to reveal the potential biological mechanisms of differentially expressed mRNAs. Then, we successfully constructed an lncRNA-related ceRNA network in EC after differential expression analysis and database comparison. Finally, univariate and multivariate $\mathrm{COX}$ regressions were used to find lncRNAs related to prognosis. Our research has found
lncRNAs related to the prognosis of EC. These lncRNAs may become markers of EC prognosis.

\section{Materials and Methods}

2.1. Data Collection and Preprocessing. RNA-seq data, microRNA data, and the clinical data of EC were downloaded from The Cancer Genome Atlas (TCGA) database (https:// cancergenome.nih.gov/). RNA-seq data contains 160 cancer samples and 11 normal samples. These data were used to identify differentially expressed $\operatorname{lncRNAs}$ and mRNAs. MicroRNA data includes 185 cancer samples and 13 normal samples. Perl (version 5.28.1; https://www.perl.org/) was used for data processing. Ensemble database (Ensemble release 99; http://asia.ensembl.org/index.html) was used for gene annotations and identification of $\operatorname{lncRNAs}$ and mRNAs. RNAs that have not been annotated by the database were excluded. Our research was conducted in accordance with TCGA publication guidelines. Therefore, the approval from the local ethics committee was not required.

2.2. Identification of Differentially Expressed RNAs. RNAs with no expression or average count $\leq 1$ will be filtered. The differentially expressed RNAs were identified using the edge $\mathrm{R}$ package in $\mathrm{R}$ (version3.5.1) [20]. | $\log 2$ fold change (FC) $\mid>1$ and false discovery rate $($ FDR $)<0.05$ were the 


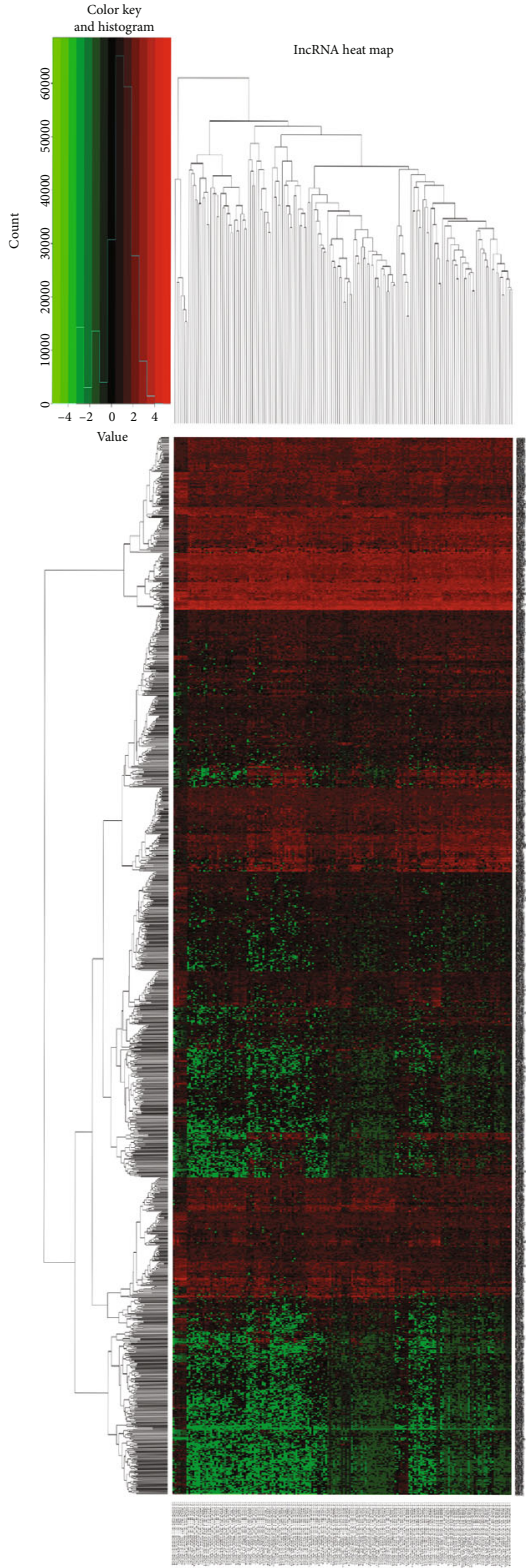

(a)
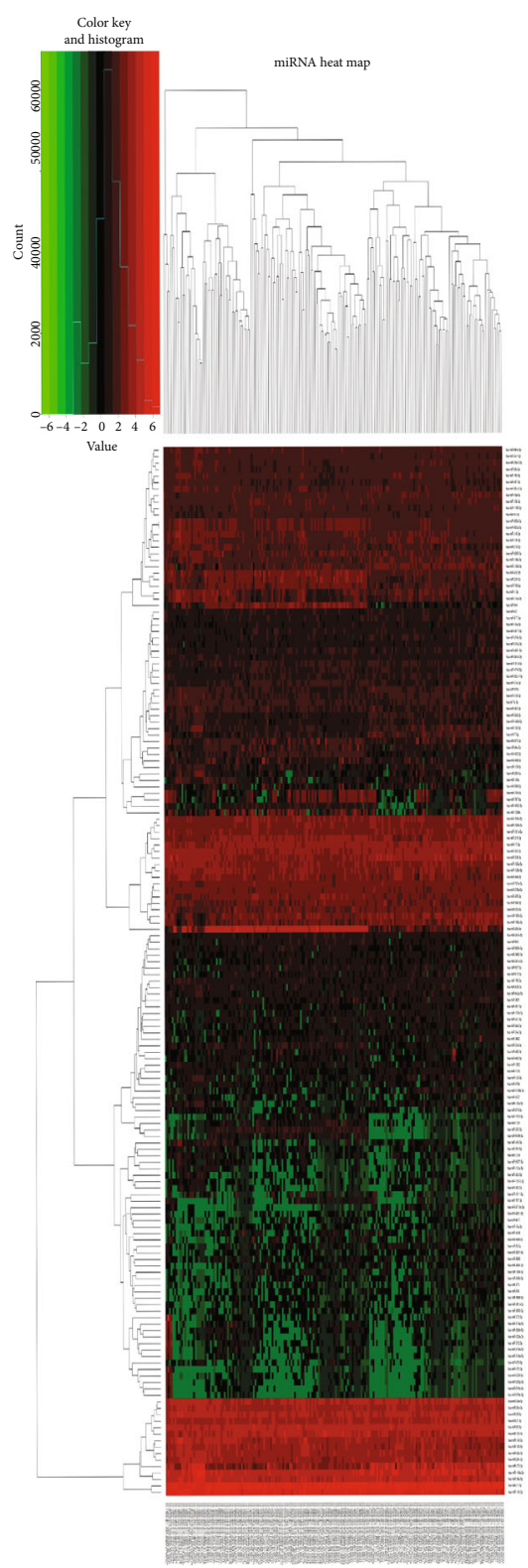

(b)
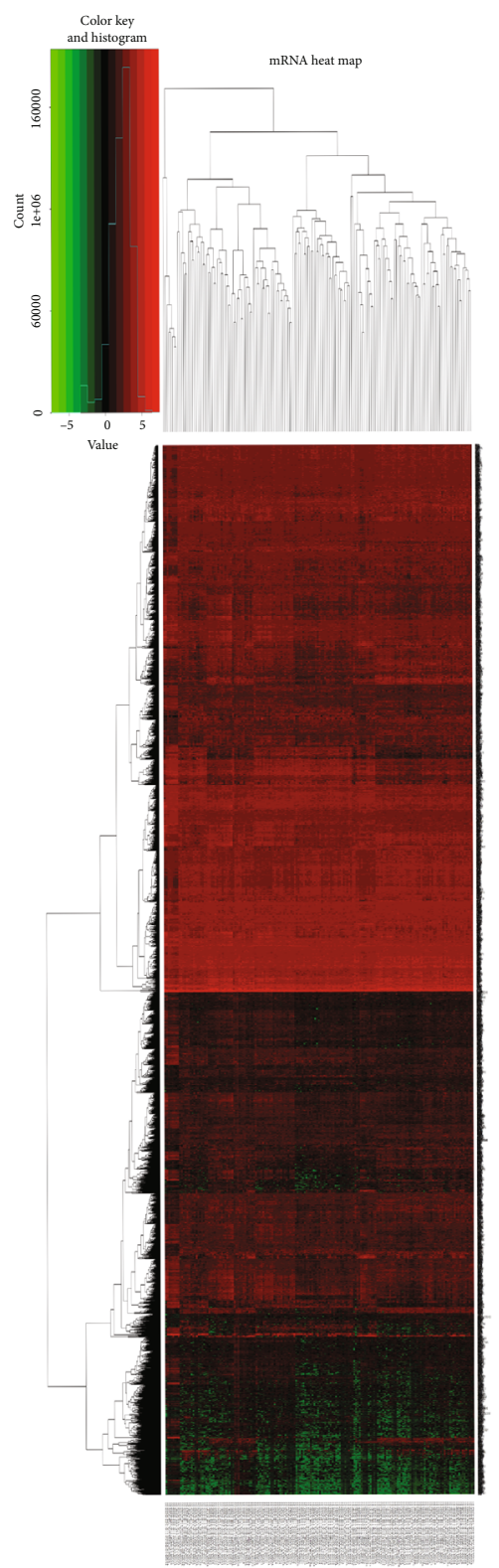

(c)

Figure 2: Heat maps of differential expression of lncRNAs, miRNAs, and mRNAs: (a) heat map of differential expression of lncRNAs; (b) heat map of differential expression of miRNAs; (c) heat map of differential expression of mRNAs.

screening condition for differential RNAs. In addition, the heat maps and volcano maps of differentially expressed RNAs were drawn by the gplots and heat map packages in the R software.

\subsection{Gene Ontology and KEGG Enrichment Analysis. DAVID} 6.8 (https://david.ncifcrf.gov) was used for Gene Ontology (GO) enrichment analysis and Kyoto Gene and Genome Encyclopedia (KEGG) signal pathway analysis based on DEmRNAs. GO enrichment analysis can classify and annotate genes through three aspects: biological path (BP), cellular component (CC), and molecular function (MF). The KEGG signaling pathway was used to find important signaling pathways.
2.4. Construction of ceRNA Network. The miRcode database (http://www.mircode.org/) was used to predict miRNAs that bind to lncRNAs. MiRTarBase (http://mirtarbase.cuhk .edu.cn/), miRDB (http://www.mirdb.org/), and TargetScan (http://www.targetscan.org/) databases were used to predict the target genes of miRNAs [21-23]. The mRNAs obtained from the overlapping parts of the three data sets and intersected with DEmRNAs were considered to be mRNAs bound to miRNAs. Then, we constructed a coexpression network of differently expressed RNAs based on the DElncRNA-DEmiRNA and DEmiRNA-DEmRNA interactions. Cytoscape (version 3.7.1) was used for network visualization. The flow chart of ceRNA network construction was displayed (see Figure 1). 


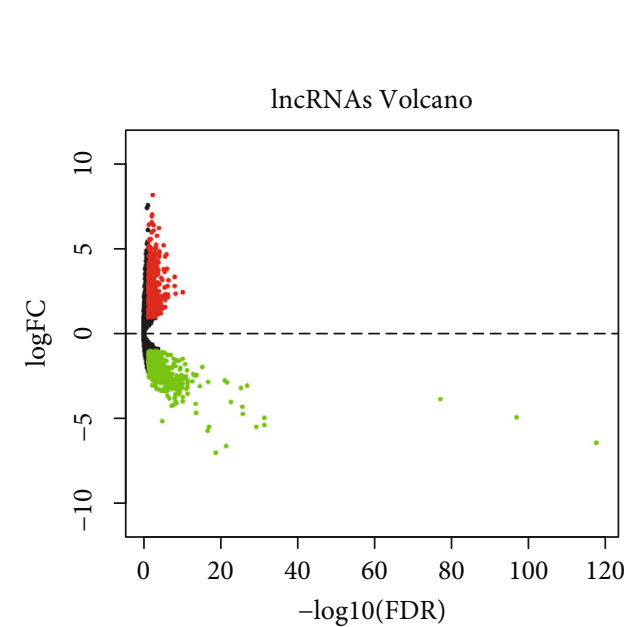

(a)

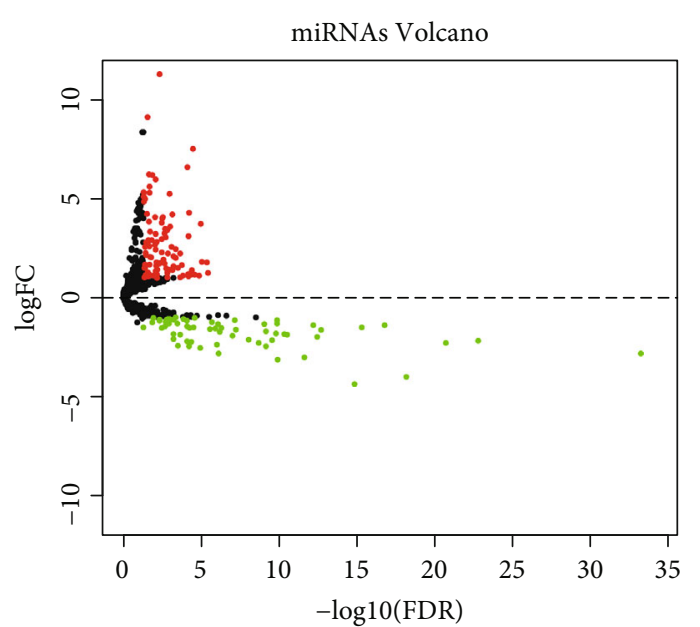

(b)

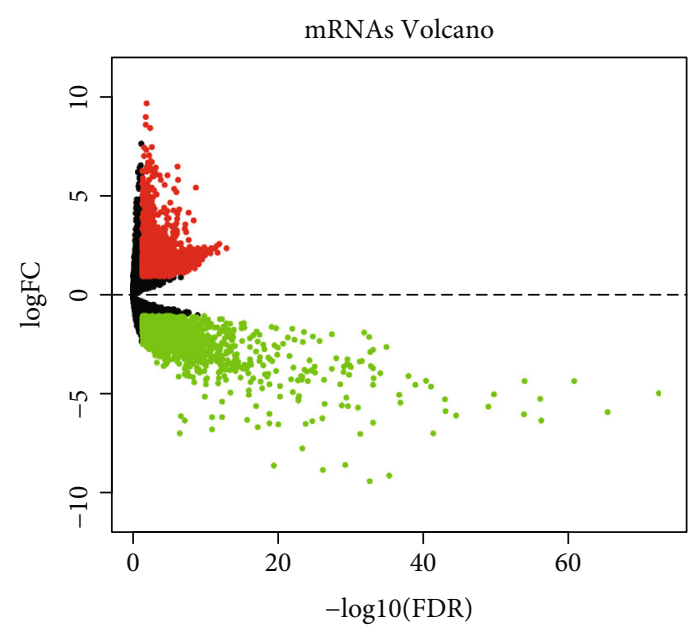

(c)

Figure 3: Volcano maps of differential expression of lncRNAs, miRNAs, and mRNAs: (a) volcano map of differential expression of lncRNAs; (b) volcano map of differential expression of miRNAs; (c) volcano map of differential expression of mRNAs. Red represents upregulated RNAs, and green represents downregulated RNAs.

2.5. Construction of PPI Network and Selection of Hub Gene. The STRING (search tool for the retrieval of interacting genes) (https://string-db.org) database was used to construct a protein interaction network for differential genes in the ceRNA network, and the medium credibility (interaction score $>0.4$ ) was the screening criterion [24]. Cytoscape (version 3.7.1) was used for PPI network visualization. The maximal clique centrality (MCC) method from the cytoHubba app in Cytoscape was used to screen for genes with higher scores, which were considered key genes $[25,26]$.

2.6. Survival Analysis and Prognostic Model. The "survival" package in $\mathrm{R}$ software was used for survival analysis of DElncRNAs in the ceRNA network. The Kaplan-Meier method was used to draw survival curves, and the log-rank test was used to compare the differences between the two groups. $p<0.05$ was considered statistically significant. Subsequently, univariate COX regression was used to evaluate the association between DElncRNAs in the ceRNA network and the overall survival rate of EC, and $p<0.05$ was consid- ered to be significant. Then, DElncRNAs from univariate cox regression and LR analysis were used to construct a multivariate Cox proportional hazards regression model. The multivariate Cox regression model was used to construct a prognostic model of DElncRNAs in the ceRNA network. The stepwise regression method was used to include and exclude variables. This method is based on Akaike Information Criterion (AIC). AIC is a standard used to measure the goodness of a statistical model. It is generally considered that the model with the smaller AIC value is the optimal model. The model expression formula is as follows:

$$
\begin{aligned}
\text { Risk score }= & \text { CoencRNA1*ExplncRNA1 } \\
& + \text { CoenlncRNA2*ExplncRNA2 } \\
& +\cdots \text { CoencRNAn } * \text { ExplncRNAn } .
\end{aligned}
$$

"Coe" represents the regression coefficient of the multiple COX regression model, and "Exp" represents the expression level of lncRNAs. 


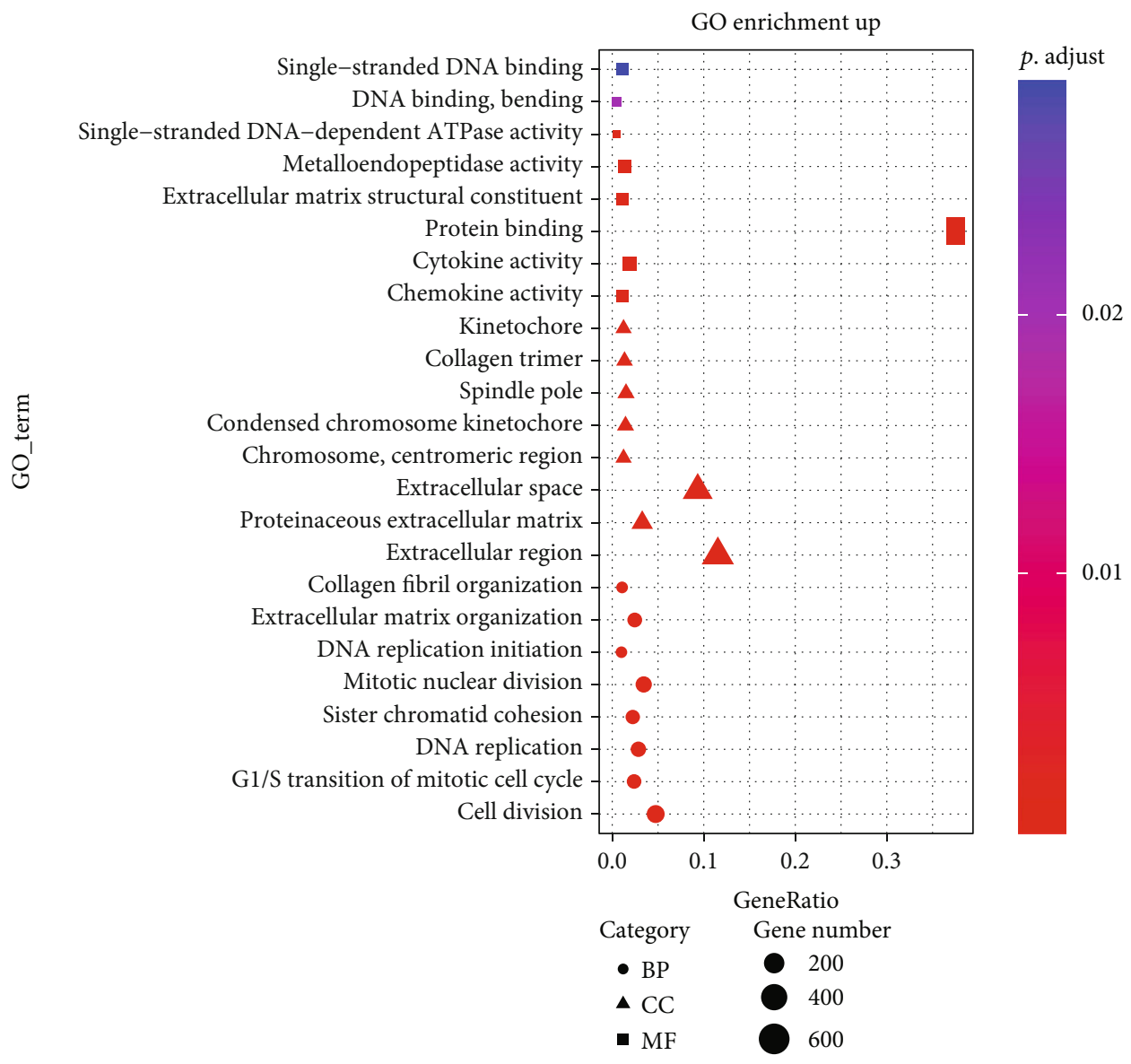

(a)

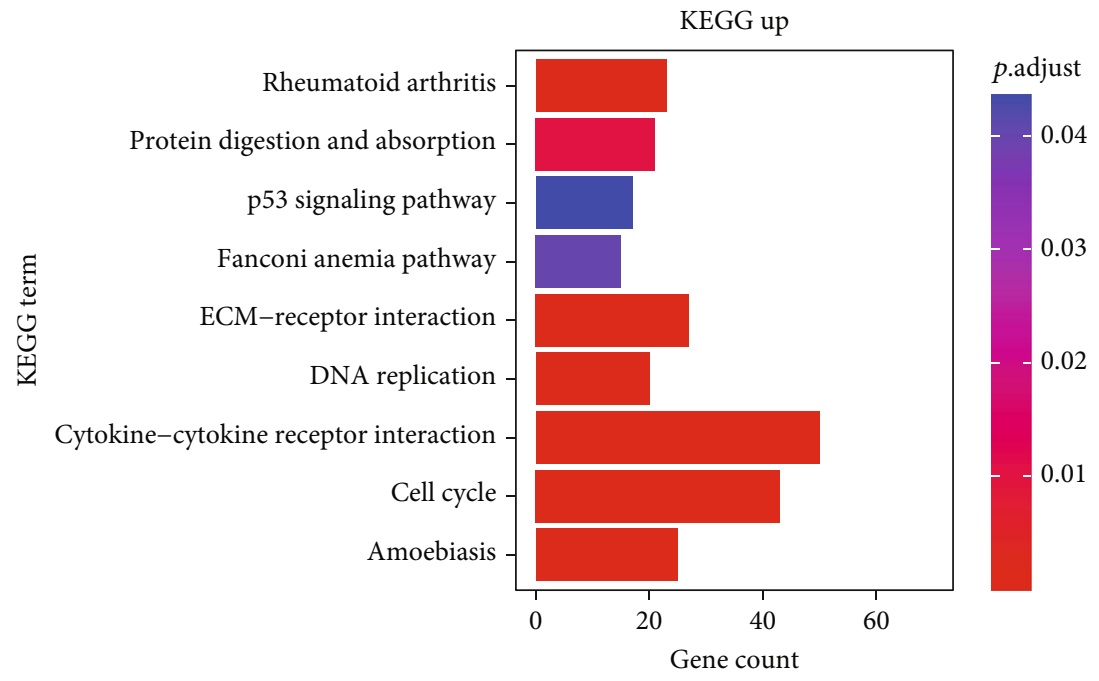

(b)

Figure 4: Continued. 


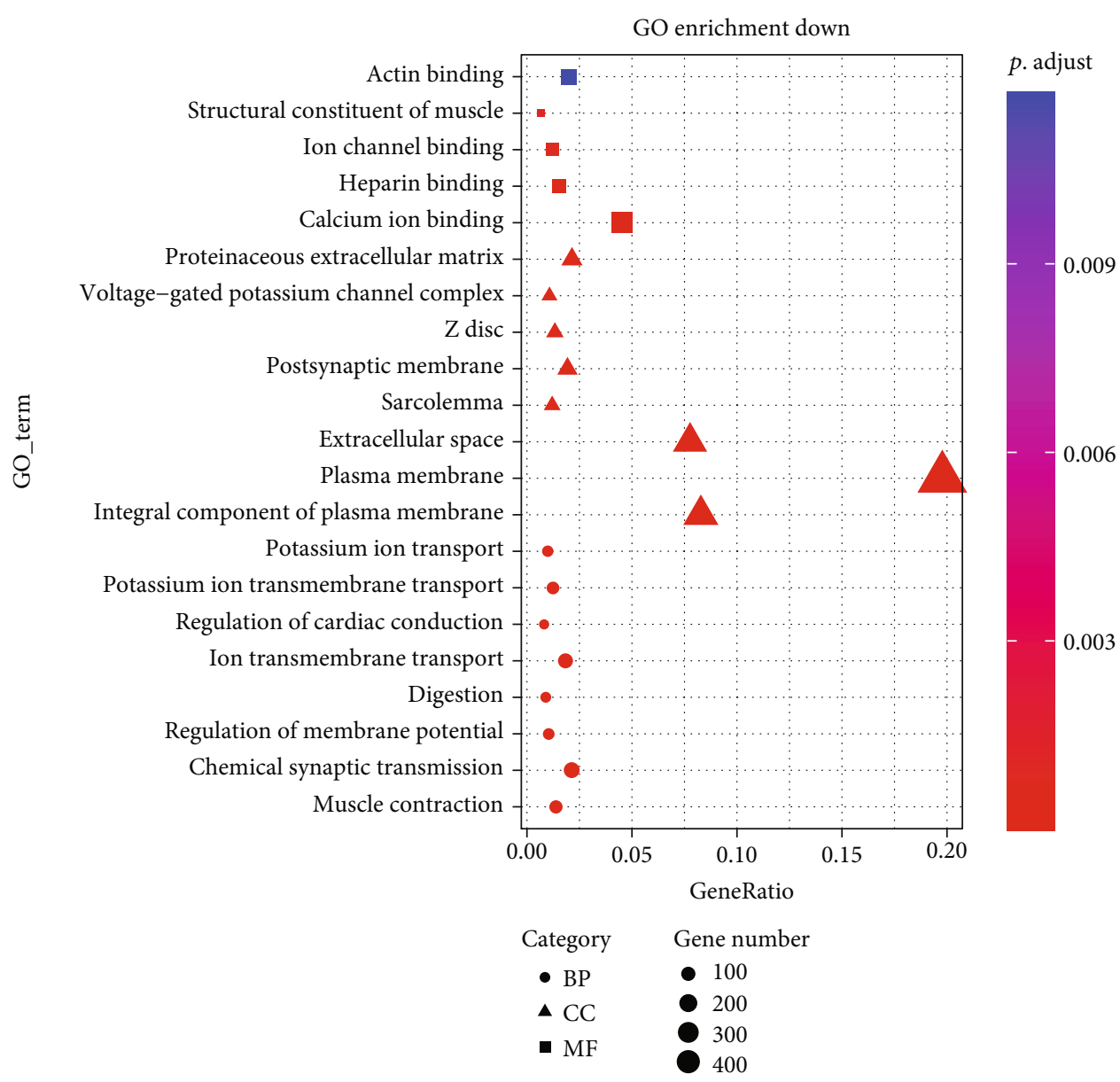

(c)

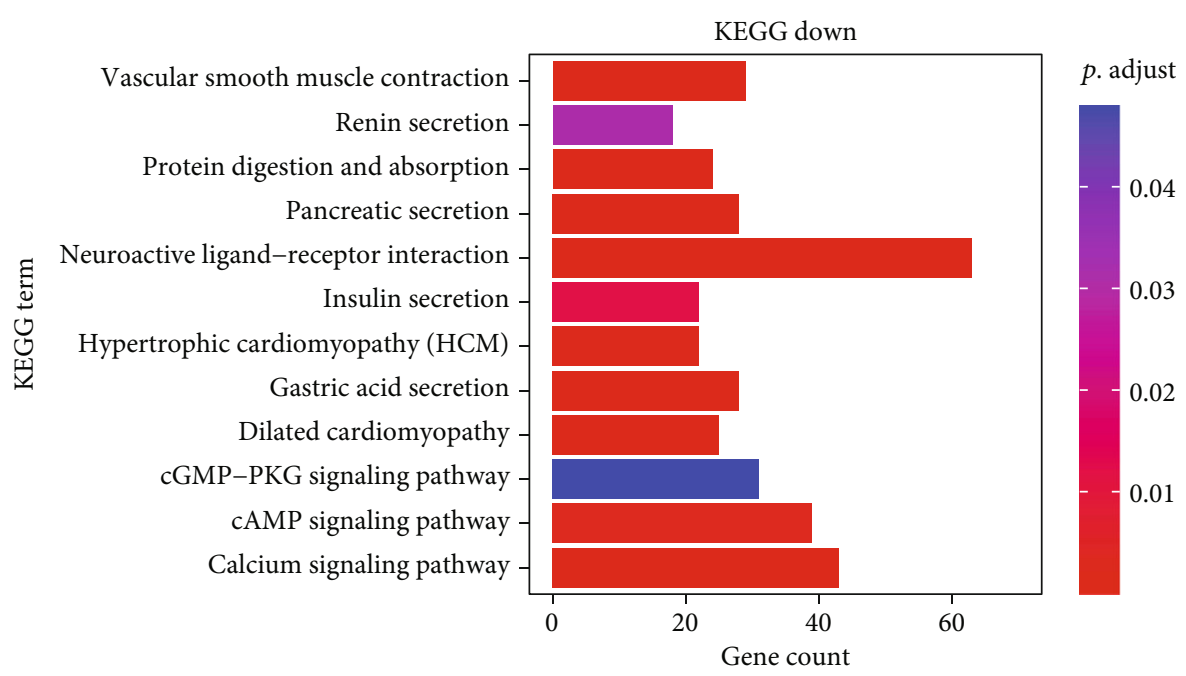

(d)

Figure 4: The gene ontology (GO) enrichment analysis of DEmRNAs and the bubble chart and bar chart of the Kyoto Encyclopedia of Gene and Genome (KEGG) signaling pathway analysis: (a) GO enrichment analysis of upregulated DEmRNAs; (b) analysis of the KEGG signaling pathway of upregulated DEmRNAs; (c) GO enrichment analysis of downregulated DEmRNAs; (d) analysis of KEGG signaling pathway of downregulated DEmRNAs.

According to the median value of risk score, EC patients were divided into high- and low-risk groups. Kaplan-Meier analysis was used to compare the overall survival rates of the two groups. The "timeROC" package was used to plot the time-dependent receiver operating characteristic (ROC) curve, which can evaluate the value of the model prediction. 
TABLE 1: DElncRNAs, DEmiRNAs, and DEmRNAs included in the ceRNA network.

\begin{tabular}{|c|c|c|}
\hline Category & Changes & Gene symbol \\
\hline \multirow[t]{2}{*}{$\operatorname{lncRNAs}$} & Downregulated & $\begin{array}{l}\text { TTTY14, C9orf106, LINC00304, WDFY3-AS2, SPATA8, C5orf60, AL162511.1, AC005082.1, LINC00269, } \\
\text { AP000897.1, AC079467.1, SNHG14, POU6F2-AS1, LINC00365, THRB-IT1, LINC00457, LINC00113, PCA3, } \\
\text { PRICKLE2-AS3, DNMBP-AS1, PCDH9-AS2, TTTY10, ZRANB2-AS2, C9orf147, DIRC3, F10-AS1, } \\
\text { MIR497HG, CYP1B1-AS1, TTLL7-IT1, LINC00472, ENOX1-AS1, JAZF1-AS1, MAGI2-AS3, ZRANB2-AS1, } \\
\text { SRGAP3-AS4, MACROD2-AS1, DAPK1-IT1,CADM2-AS1, ZBTB20-AS3, MAGI-AS1, ADAMTS9-AS1, } \\
\text { ADAMTS9-AS2, AL353803.1, AC114810.1, AC021755.3, AC018926.1, AP001094.1, AC007389.1, FOXP1-AS1, } \\
\text { AL391807.1, AP005717.1, AC012181.1, LIFR-AS1, ALDH1L1-AS2, FAM13A-AS1, DNAH10OS, C8orf49, } \\
\text { AC110619.1, RMST, AC135776.1, PWRN1, LINC00261 }\end{array}$ \\
\hline & Upregulated & $\begin{array}{l}\text { PVT1, DLEU2, SNHG1, LINC00460, GK-IT1, ALMS1-IT1, LINC00337, SNHG15, ZEB1-AS1, SNHG3, } \\
\text { POU6F2-AS2, AL391152.1, AC007611.1, C17orf82, LINC00184, HOTAIR, LINC00392, C15orf54, AP002478.1, } \\
\text { C8orf31, TM4SF19-AS1, HCP5, AC009093.1, AL513123.1, DLX6-AS1, FNDC1-IT1, LINC00393, AC131254.1, } \\
\text { LINC00355, AL118508.1, AC131157.1, CASK-AS1, LMO7-AS1, AC093515.1, AC019294.2, HOTAIRM1, } \\
\text { AC004917.1, NAALADL2-AS2, AC123768.1, MYO16-AS1, LINC00299, LINC00491, LINC00114, DSCR8, } \\
\text { LSAMP-AS1, LINC00237, E2F3-IT1, LGALS8-AS1, KTN1-AS1 }\end{array}$ \\
\hline \multirow[t]{2}{*}{ miRNAs } & Downregulated & $\begin{array}{l}\text { hsa-miR-139-5p, hsa-miR-338-3p, hsa-miR-125a-5p, hsa-miR-125b-5p, hsa-miR-129-5p, hsa-miR-490-3p, } \\
\text { hsa-miR-363-3p, hsa-miR-135a-5p }\end{array}$ \\
\hline & Upregulated & hsa-miR-17-5p, hsa-miR-301b-3p, hsa-miR-508-3p \\
\hline \multirow{2}{*}{ mRNAs } & Downregulated & $\begin{array}{l}\text { NR3C2, VLDLR, TXNIP, CADM2, ADAMTSL3, KAT2B,RBM47, RBPMS2, GRM7, DBT, SCRG1, PTF1A, } \\
\text { NFIC, CNTN4, DCLK2, ZNF385B, GLUL, LPP, SATB1, TTC28, THRB, NTN4, RGMB, CRY2, STARD13, } \\
\text { GAB2, LIFR, FAM174B, LIN28A, BTG2, GATA6, NOVA1, PDE4D }\end{array}$ \\
\hline & Upregulated & $\begin{array}{l}\text { MMP11, EZH2, CBFB, BCL2L12, E2F3, DUSP10, HAUS8, CENPQ, COL1A1, ZNF367, ADAM17, CEP19, } \\
\text { RUNX2, TNFAIP3, HOXD10, SEMA7A, ASPN, FJX1, DUSP6, HMGA2, COL5A1, ARID3A, CAMK2N2, } \\
\text { EIF4EBP1, MSN, STON2, EGR2, HOXA10, ETV1, NEUROD2 }\end{array}$ \\
\hline
\end{tabular}

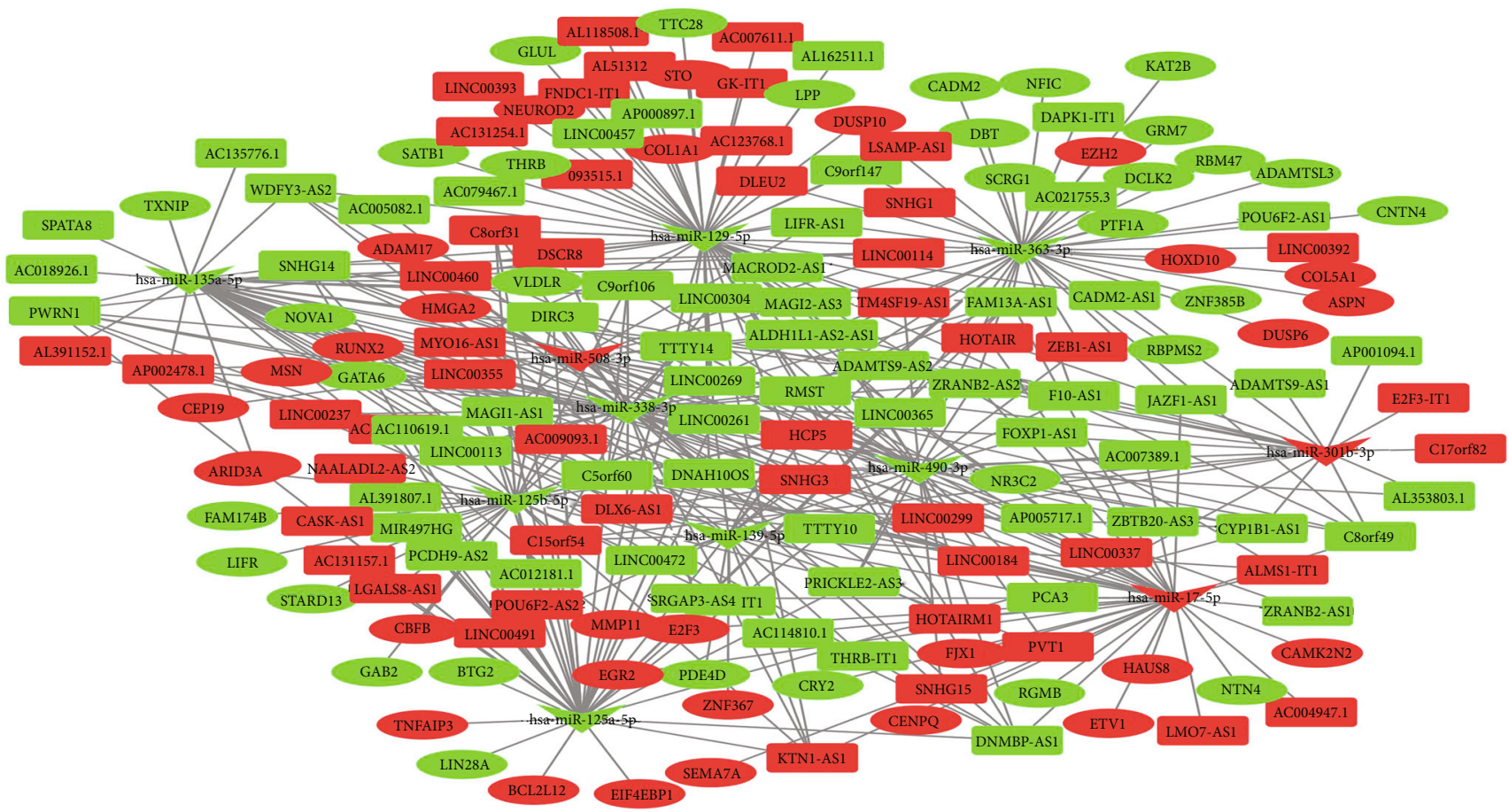

FIGURE 5: the ceRNA network of lncRNA-miRNA-mRNA in EC. Rectangles represent lncRNAs, V represents miRNAs, and ellipses represent mRNAs. The red nodes are upregulated RNAs, and the green nodes are downregulated RNAs.)

\section{Results}

3.1. Identification of the DElncRNAs, DEmiRNAs, and $D E m R N A s$. The differentially expressed RNAs were identified by using the edgeR package in $\mathrm{R}$ software. $\mid \log 2$ fold change $(\mathrm{FC}) \mid>1$ and false discovery rate $(\mathrm{FDR})<0.05$ were the screening condition for differential RNAs. 1331 DElncRNAs (648 downregulated and 683 upregulated) and 3193 DEmRNAs (1753 downregulated and 1440 upregulated) were screened out of 160 EC samples and 11 normal samples. 


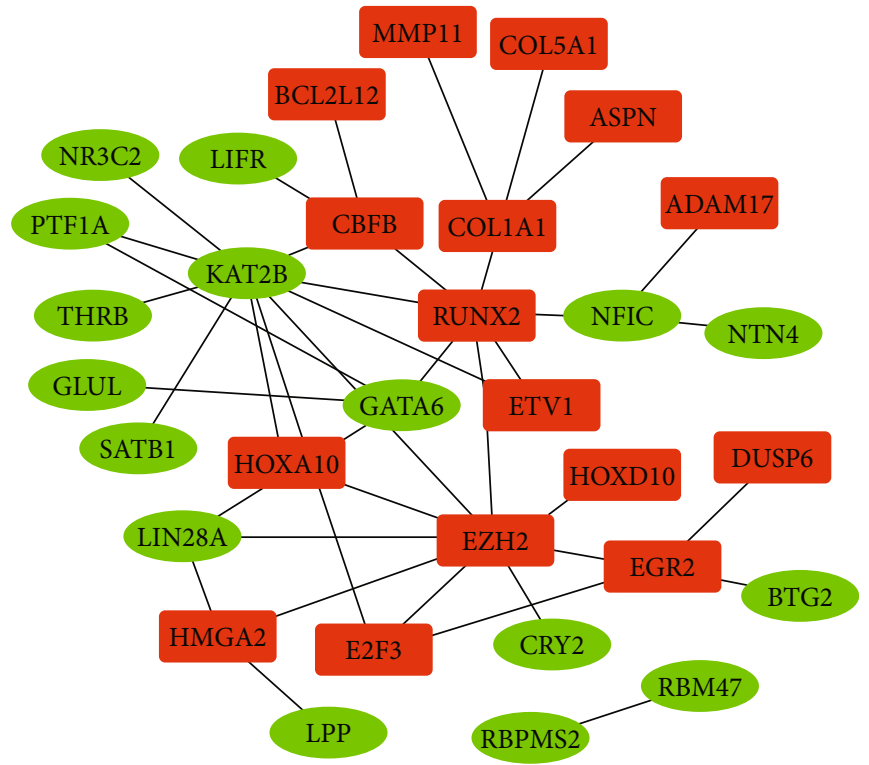

(a)

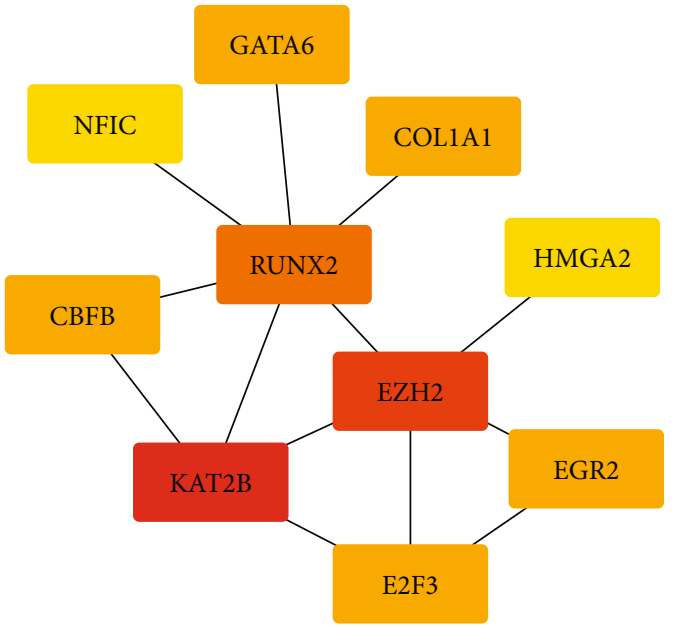

(b)

FIGURE 6: Identification of hub genes from the PPI network with the MCC method. (a) There are 32 genes in the PPI network. The red nodes are upregulated genes, and the green nodes are downregulated genes. (b) Top ten key genes screened by the MCC method; red was the higher score calculated by the MCC method, followed by orange.

162 DEmiRNAs (64 downregulated and 98 upregulated) were screened out of 185 cancer samples and 13 normal samples. The heat map of differential RNAs is displayed (see Figure 2). The volcano diagram of differential RNAs is displayed (see Figure 3). The results indicated that these differential RNAs might play a role in the development of EC.

3.2. Functional Analysis of DEmRNAs. DAVID 6.8 (https:// david.ncifcrf.gov) was used for Gene Ontology (GO) enrichment analysis and Kyoto Gene and Genome Encyclopedia (KEGG) signal pathway analysis based on DEmRNAs. GO enrichment analysis can classify and annotate genes through three aspects: biological path (BP), cellular component (CC), and molecular function (MF). Upregulated mRNAs were classified as 47 biological process (BP) terms, 20 cellular component (CC) terms, and 8 molecular function (MF) terms using GO enrichment analysis. Downregulated mRNAs were classified as 10 biological process (BP) terms, 12 cellular component (CC) terms, and 5 molecular function (MF) terms using GO enrichment analysis. The results of partial GO enrichment are displayed (see Figures 4(a) and 4(c)). Subsequently, KEGG signaling pathway analysis showed that upregulated mRNAs were significantly enriched in 9 signaling pathways, and downregulated mRNAs were significantly enriched in 12 signaling pathways (see Figures 4(b) and $4(d)$ ). The upregulated mRNAs were mainly involved in cell cycle, DNA replication, cytokine-cytokine receptor interaction, and ECM-receptor interaction. Downregulated mRNAs were mainly involved in neuroactive ligand-receptor interaction, gastric acid secretion, calcium signaling pathway, and pancreatic secretion. These results can help us understand the key signaling pathways and biological processes in the development of EC.
TABLE 2: MCC method to calculate the key genes and their scores in the PPI network.

\begin{tabular}{lcc}
\hline Rank & Gene symbol & Score \\
\hline 1 & KAT2B & 14 \\
2 & EZH2 & 12 \\
3 & RUNX2 & 9 \\
4 & COL1A1 & 4 \\
5 & E2F3 & 4 \\
6 & CBFB & 4 \\
7 & EGR2 & 4 \\
8 & GATA6 & 4 \\
\hline
\end{tabular}

3.3. Construction of ceRNA Network. Bioinformatics tools were used to predict the interaction between DElncRNAs, DEmiRNAs, and DEmRNAs. The miRcode database (http://www.mircode.org/) was used to predict miRNAs that bind to lncRNAs. The Perl language was used to extract DElncRNAs and miRNAs combined with DElncRNAs in the miRcode database. Then, the DEmiRNAs and the miRNAs extracted from the miRcode database were intersected to obtain the miRNAs in the ceRNA network. The lncRNAs targeted by these miRNAs were the lncRNAs in the ceRNA. MiRTarBase, miRDB, and TargetScan databases were used to predict the target genes of miRNAs in the ceRNA network. After these target genes intersect with DEmRNAs, they were the mRNAs in the ceRNA network. In the end, 111 lncRNAs (62 downregulated and 49 upregulated), 11 miRNAs (8 downregulated and 3 upregulated), and 63 mRNAs (33 downregulated and 30 upregulated) were included in the ceRNA network. The lncRNAs, miRNAs, and mRNAs in the ceRNA network are displayed (see 

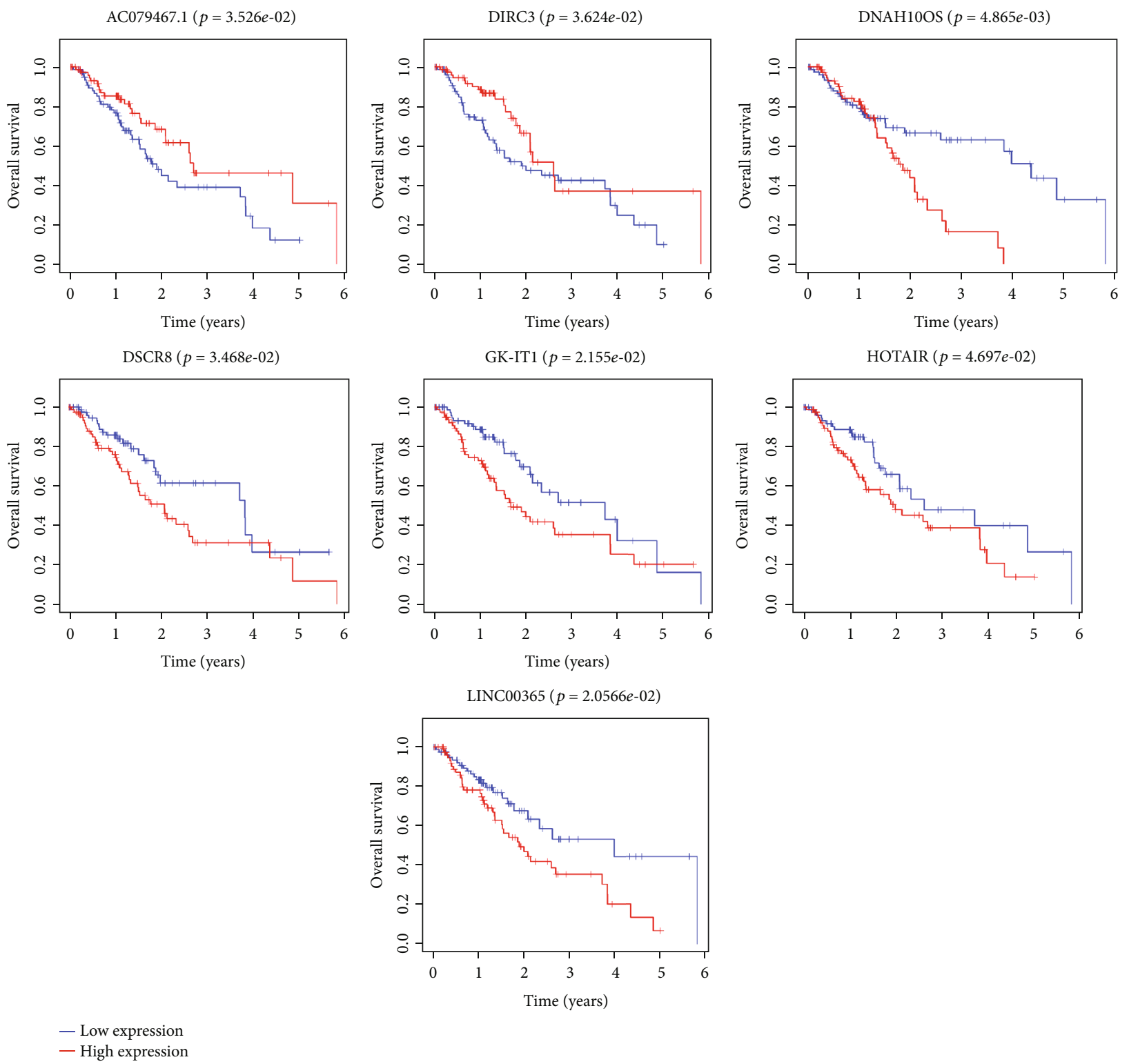

FIGURE 7: Kaplan-Meier survival curves of seven DElncRNAs associated with overall survival in EC.

Table 1). Cytoscape was used for network visualization. ECrelated ceRNA network is displayed (see Figure 5).

\subsection{Construction of PPI Network and Selection of Hub Gene.} The STRING (search tool for the retrieval of interacting genes) database (https://string-db.org) was used to construct a protein interaction network of differential genes. Medium credibility (interaction score $>0.4$ ) was used as the screening criterion, loose links and outliers were removed, and the PPI network was drawn. Cytoscape was used for PPI network visualization. The PPI network is displayed (see Figure 6(a)). The maximal clique centrality (MCC) method from the cytoHubba app in Cytoscape was used to screen for genes with higher scores, which were considered key genes. The key genes are displayed (see Figure 6(b), Table 2). Our results indicated that KAT2B, EZH2, RUNX2, COL1A1, E2F3, CBFB, EGR2, GATA6, NFIC, and HMGA2 had important roles in the ceRNA network. These genes might be key genes in the development of EC.

3.5. Survival Analysis and Prognostic Model Construction. More and more evidences have shown that lncRNAs can predict the overall survival rate of cancer patients. We identified lncRNAs related to prognosis from related DElncRNAs in the ceRNA network. The Kaplan-Meier method was used to draw survival curves, and log-rank test was used to compare the differences between the two groups. Seven lncRNAs were considered to be related to the overall survival rate of EC (see Figure 7). AC079467.1 and DIRC3 were considered to be positively correlated with overall survival. DNAH10OS, DSCR8, GK-IT1, HOTAIR, and LINC00365 were considered to be negatively correlated with overall survival. Then, univariate cox regression analysis was used for further analysis. Our results showed that only three lncRNA univariate cox 


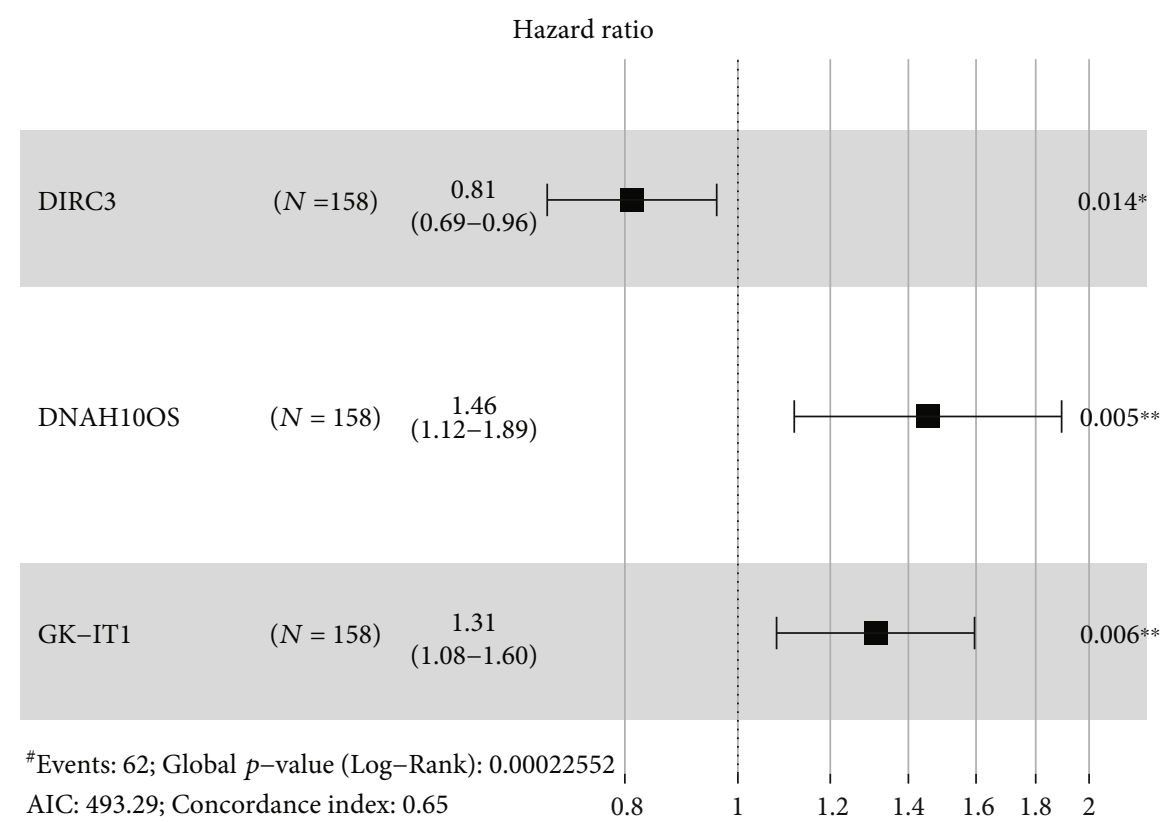

(a)

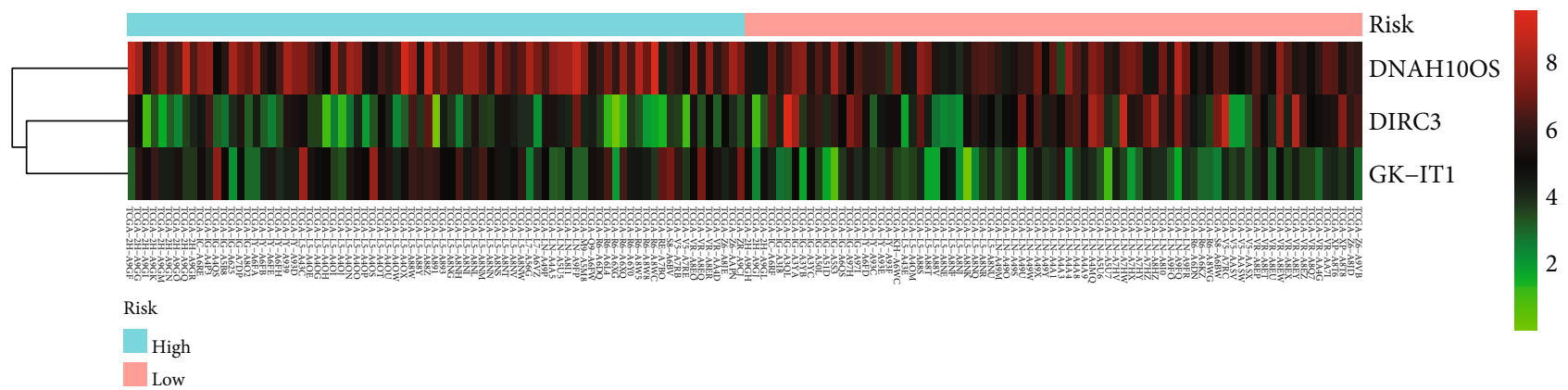

(b)

FIGURE 8: The prognostic model containing 3 lncRNAs was constructed by multiple Cox regression. (a) The prognostic model contains a forest plot of hazard ratios of three lncRNAs. (b) Heat map of the expression levels of the three lncRNAs included in the model in the high- and low-risk groups.

regression results were statistically significant in seven lncRNAs. Multivariate cox regression was used to construct a prognostic model related to lncRNAs. All three lncRNAs were included in the model. Among them, DIRC3 might be a protective factor for the prognosis of OS $(\mathrm{HR}<1$, see Figure $8(\mathrm{a}))$. DNAH10OS and GK-IT1 might be risk factors for the prognosis of OS ( $\mathrm{HR}>1$, see Figure 8(a)). The model expression formula is as follows:

$$
\begin{aligned}
\text { Risk score }= & -0.20910 * \operatorname{Exp}_{\mathrm{DIRC} 3}+0.37509 \\
& * \operatorname{Exp}_{\mathrm{DNAH} 10 \mathrm{OS}}+0.27170 * \operatorname{Exp}_{\mathrm{GK}-\mathrm{IT} 1}
\end{aligned}
$$

According to the median value of risk score, EC patients were divided into high- and low-risk groups. The heat map of the expression profiles of the three lncRNAs in EC patients is displayed (see Figure 8(b)).

Among them, there were 79 samples of EC patients in the high-risk group and the low-risk group respectively. In order to reflect the predictive performance of the model, $\mathrm{K}-\mathrm{M}$ analysis was used to compare the overall survival rate of the highrisk group and the low-risk group. The results showed that the overall survival rate between the two groups was statistically different (see Figure 9(c), $p<0.05$ ). The calibration curve was used to test the consistency between the model's predicted mortality rate and the actual mortality rate. The calibration curves of the 3-year overall survival rate (see Figure 9(a)) showed that there was good agreement between the predicted mortality rate and the actual mortality rate. In addition, our model was used to predict the 3-year survival rate and 5-year survival rate of patients with EC. The timedependent receiver operating characteristic (ROC) curves are displayed (see Figure 9(b)). The area under the curve for 3-year survival rate was 0.639 , and the area under the curve for 5-year survival rate was 0.685 . These results indicated that the PI of lncRNAs showed a good prognostic ability, which suggested that the prognostic model constructed by the three lncRNAs may be a prognostic factor for EC. 


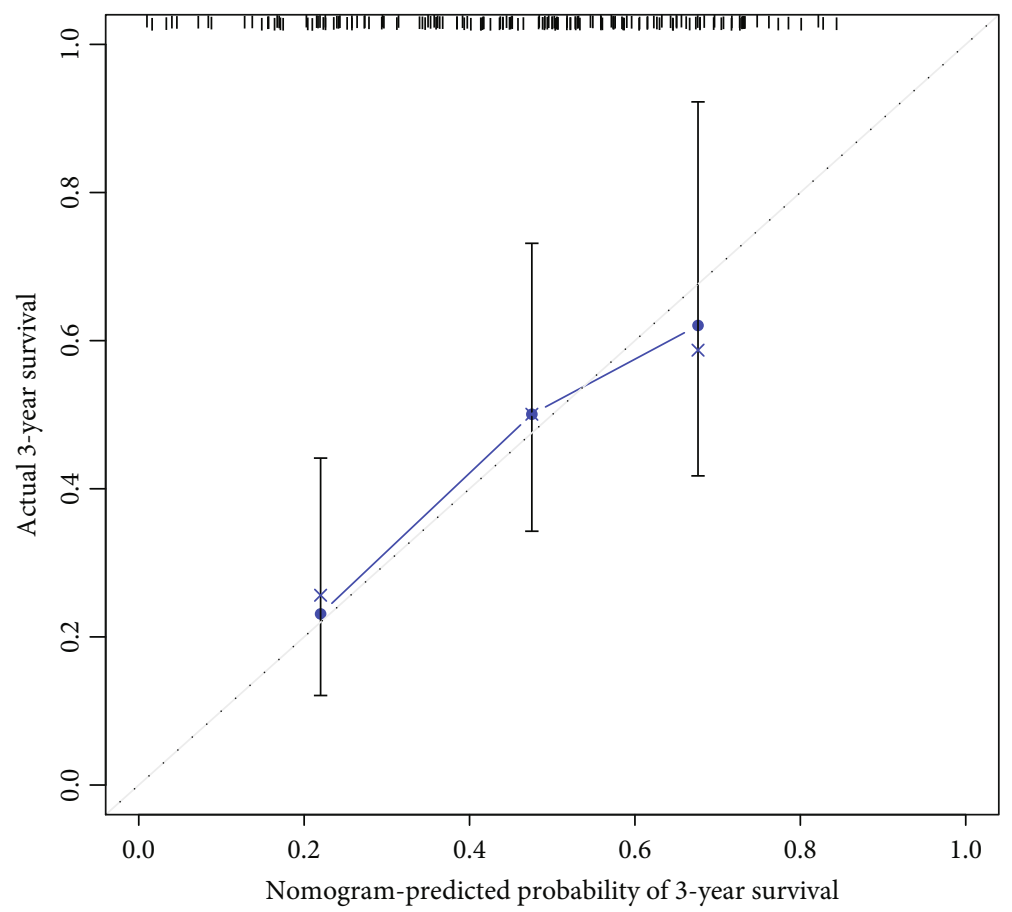

(a)

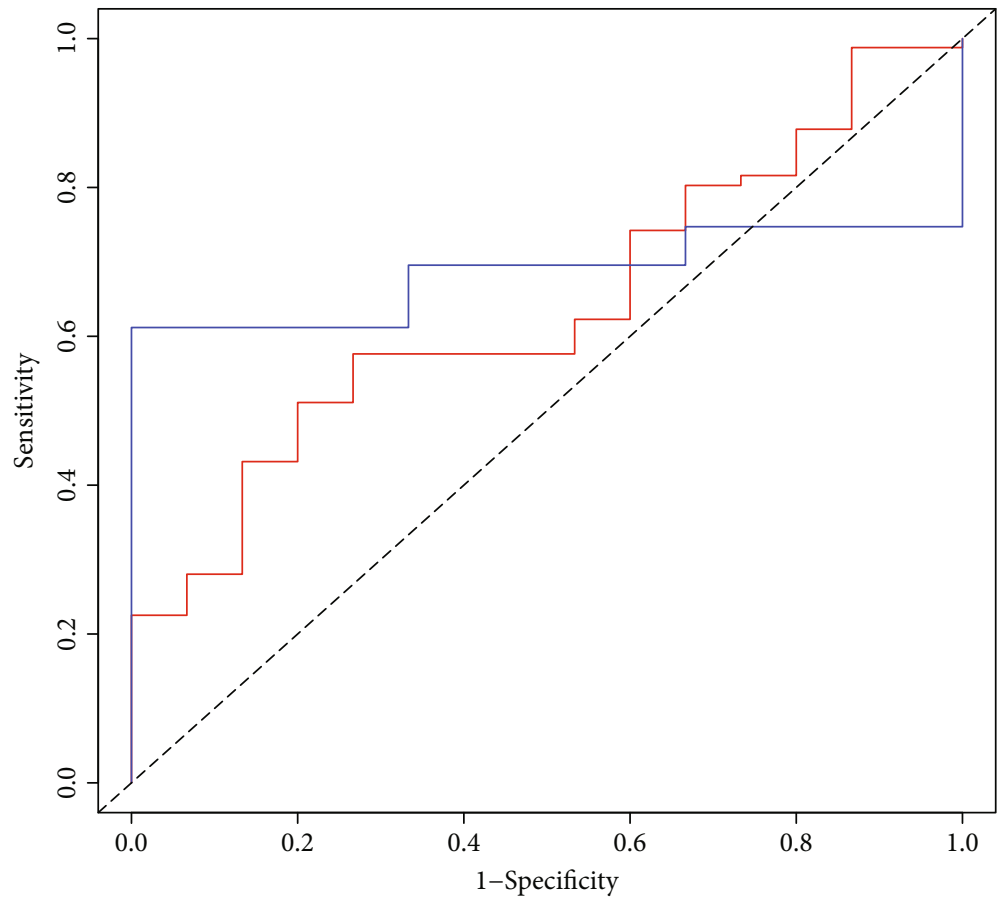

- AUC of 3 year survival: 0.639

- AUC of 5 year survival: 0.685

(b)

Figure 9: Continued. 


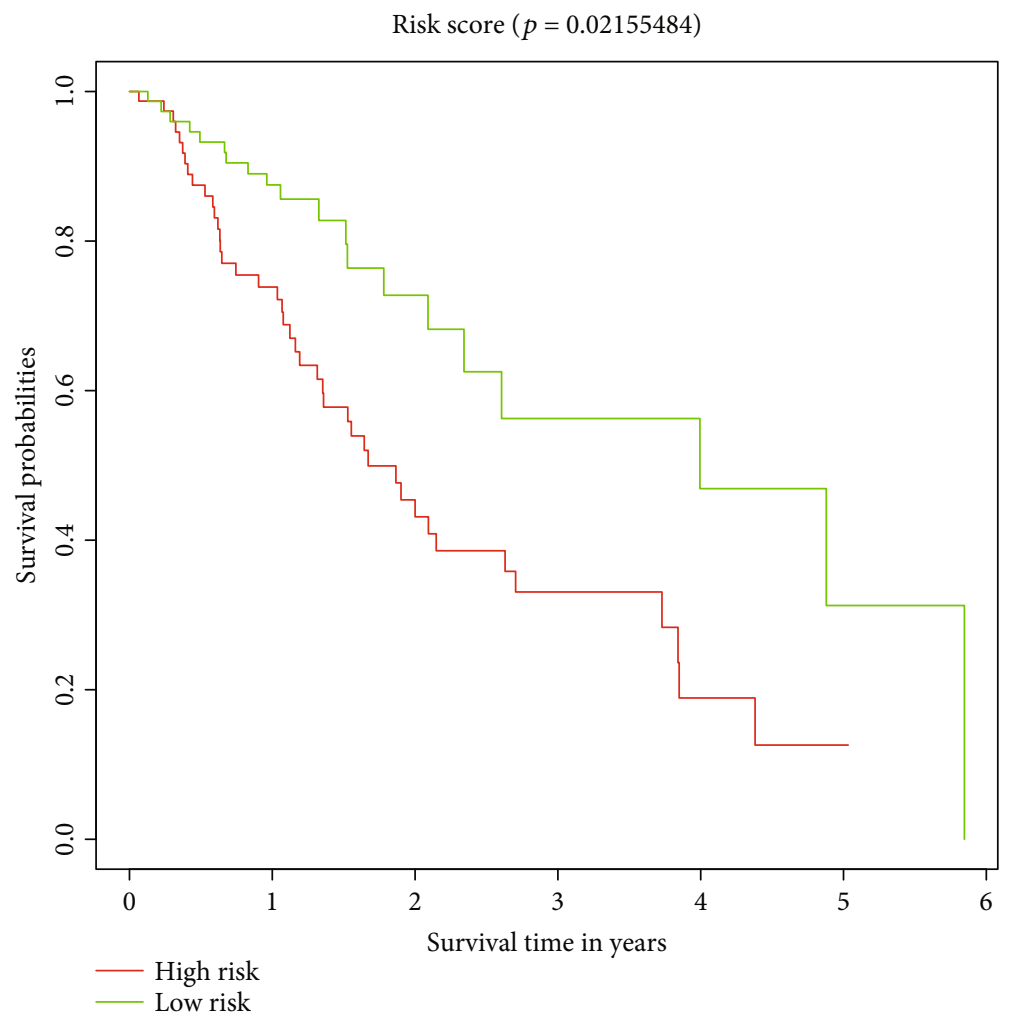

(c)

Figure 9: Evaluation of model prognostic ability. (a) The calibration curves of the 3-year overall survival rate; the abscissa was the predicted mortality of the model, and the ordinate was the actual mortality. (b) The receiver operating characteristic (ROC) curve of the three-year survival rate and the five-year survival rate predicted by the model. (c) Kaplan-Meier plots of overall survival for patients with a high- or low-risk score of the EC patients.

\section{Discussion}

Esophageal cancer (EC) is one of the most deadly malignant tumors. The five-year survival rate of EC is only $15 \%-25 \%$ [4-6]. Therefore, there is an urgent need to find molecular biomarkers for EC, which can help improve the prognosis and treatment of patients with EC. Long noncoding RNA has been confirmed to be associated with poor prognosis in lung cancer [27, 28], gastric cancer [29, 30], liver cancer [31], and other cancers [32].

lncRNAs can regulate the occurrence of diseases in a variety of ways. Existing studies have shown that lncRNAs can interact with epigenetics to regulate disease processes in the nucleus; these interactions include the interaction between lncRNAs and DNA methylation [33]. IncRNAs can also interact with transcription factors to play an important role at the transcription level [34]. IncRNAs can directly bind to mRNA to increase its stability or make it degrade, inhibit, or promote its translation in the cytoplasm [18]. However, more research has focused on lncRNAs as a member of the ceRNA network to play its regulatory role, which has even become the research focus of lncRNAs in diseaserelated roles.

More and more evidence has shown that lncRNAs participate in the regulation of EC through the ceRNA network mechanism. IncRNA MALAT1 can regulate miR-101 and miR-207 to affect the proliferation, invasion, and metastasis of ESCC cells [11]. SNHG16, which is upregulated in ESCC tissues and cell lines, can bind miR-140-5p to regulate the expression of ZEB1 [35]. The expression of lncRNA HAND2-AS1 is downregulated in tumor tissue. The overexpression of IncRNA HAND2-AS1 may inhibit the proliferation, migration, and invasion of cancer cells in ESCC by downregulating miRNA-21 [36]. These studies indicated that IncRNAs can play a role through the ceRNA network in EC, and lncRNAs are closely related to the occurrence of tumors. Therefore, our study screened differentially expressed lncRNAs, miRNAs, and mRNAs in EC through TCGA database. Subsequently, we constructed 111 lncRNAs (62 downregulated and 49 upregulated), 11 miRNAs (8 downregulated and 3 upregulated), and 63 mRNAs (33 downregulated and 30 upregulated) were included in the ceRNA network. Here, we are concerned about the relative regulation of lncRNAs in the ceRNA network. We have constructed the PPI network and found the key genes in the network. Our results indicated that KAT2B, EZH2, RUNX2, COL1A1, E2F3, CBFB, EGR2, GATA6, NFIC, and HMGA2 had important roles in the ceRNA network. These genes may play an important role in EC [3740]. Here, we identified lncRNAs related to prognosis from related DElncRNAs in the ceRNA network. Seven lncRNAs are considered to be related to the prognosis of EC. HOTAIR has been confirmed to have higher expression levels in ESCC tissues than in the corresponding noncancerous tissues in 
these seven lncRNAs. Increased HOTAIR expression is related to poor prognosis. In the clinical cohort study, it was also found that HOTAIR has corresponding prognostic value in ESCC $[13,14]$. DSCR8 has been shown to be involved in the progression of cancer. DSCR8 can activate the $\mathrm{Wnt} / \beta$-catenin signaling pathway to promote HCC progression through the DSCR8/miR-485-5p/FZD7 axis [41]. Similarly, DSCR8 has been shown to be dysregulated in ovarian cancer [42]. In our study, the upregulation of DSCR8 changes significantly, which means that it may play an important role in the occurrence of EC. DIRC3 is upregulated in melanoma and may be used as an inhibitor of melanoma. But DIRC3 has not been shown to be significantly different in EC [43]. LINC00365 is upregulated in colorectal cancer specimens, and it may be involved in the process of CRC cells by mediating the $\mathrm{Wnt} / \beta$-catenin pathway [44]. However, the expression of LINC00365 was downregulated in our study, which may be caused by different cancer types. The other three lncRNAs have not been reported; they may be newly discovered lncRNAs.

Subsequently, we constructed a prognostic model of EC through univariate and multivariate cox regression models. Three lncRNAs were included in the model. The prognostic model constructed by these three lncRNAs showed good predictive ability.

\section{Conclusions}

Our research is based on TCGA database to screen differentially expressed $\operatorname{lncRNAs,~miRNAs,~and~mRNAs~in~esopha-~}$ geal cancer. Then, the ceRNA network containing lncRNAs was constructed, which was used to find seven IncRNAs that related to the prognosis of esophageal cancer. Finally, a prognostic model of esophageal cancer containing three lncRNAs was constructed. These lncRNAs may be used as prognostic markers of esophageal cancer. In conclusion, our research will provide new insights into the regulation of lncRNAs in esophageal cancer.

\section{Data Availability}

The Cancer Genome Atlas (TCGA) database (https:// cancergenome.nih.gov/) is used to provide RNA-seq data, microRNA data, and the clinical data of EC.

\section{Conflicts of Interest}

The authors declare that they have no conflicts of interest.

\section{Authors' Contributions}

The download and analysis of the data are completed by Chao Li. Chao Li wrote the first draft of the paper. Changfu $\mathrm{Hao}$ and $\mathrm{Wu}$ Yao revised the first draft of the article. All authors were involved in the study and approved the final version of the manuscript.

\section{Acknowledgments}

This research was funded by the National Natural Science Foundation of China (81773404).

\section{Supplementary Materials}

Supplementary File 1: the R code and the corresponding running results to build the multiple COX regression model. (Supplementary Materials)

\section{References}

[1] F. Bray, J. Ferlay, I. Soerjomataram, R. L. Siegel, L. A. Torre, and A. Jemal, "Global cancer statistics 2018: GLOBOCAN estimates of incidence and mortality worldwide for 36 cancers in 185 countries," CA: a Cancer Journal for Clinicians, vol. 68, no. 6, pp. 394-424, 2018.

[2] A. K. Rustgi and H. B. El-Serag, "Esophageal carcinoma," The New England Journal of Medicine, vol. 371, no. 26, pp. 24992509, 2014.

[3] R. L. Siegel, K. D. Miller, and A. Jemal, "Cancer statistics, 2015," CA: A Cancer Journal for Clinicians, vol. 65, no. 1, pp. 5-29, 2015.

[4] A. Pennathur, M. K. Gibson, B. A. Jobe, and J. D. Luketich, "Oesophageal carcinoma," Lancet, vol. 381, no. 9864, pp. 400-412, 2013.

[5] G. F. Le Bras, M. H. Farooq, G. W. Falk, and C. D. Andl, "Esophageal cancer: the latest on chemoprevention and state of the art therapies," Pharmacological Research, vol. 113, Part A, pp. 236-244, 2016.

[6] S. Pasquali, G. Yim, R. S. Vohra et al., "Survival after neoadjuvant and adjuvant treatments compared to surgery alone for resectable esophageal carcinoma a network meta-analysis," Annals of Surgery, vol. 265, no. 3, pp. 481-491, 2017.

[7] J. S. Mattick and I. V. Makunin, "Non-coding RNA," Human Molecular Genetics, vol. 15, Supplement_1, pp. R17-R29, 2006.

[8] R. Spizzo, M. I. Almeida, A. Colombatti, and G. A. Calin, "Long non-coding RNAs and cancer: a new frontier of translational research?," Oncogene, vol. 31, no. 43, pp. 4577-4587, 2012.

[9] X. Huang, X. Zhou, Q. Hu et al., “Advances in esophageal cancer: a new perspective on pathogenesis associated with long non-coding RNAs," Cancer Letters, vol. 413, pp. 94-101, 2018.

[10] P. J. Batista and H. Y. Chang, "Long noncoding RNAs: cellular address codes in development and disease," Cell, vol. 152, no. 6, pp. 1298-1307, 2013.

[11] X. Wang, M. Li, Z. Wang et al., "Silencing of long noncoding RNA MALAT1 by miR-101 and miR-217 inhibits proliferation, migration, and invasion of esophageal squamous cell carcinoma cells," The Journal of Biological Chemistry, vol. 290, no. 7, pp. 3925-3935, 2015.

[12] H. L. Luo, M. D. Huang, J. N. Guo et al., "AFAP1-AS1 is upregulated and promotes esophageal squamous cell carcinoma cell proliferation and inhibits cell apoptosis," Cancer Medicine, vol. 5, no. 10, pp. 2879-2885, 2016.

[13] X. S. Ge, H. J. Ma, X. H. Zheng et al., "HOTAIR, a prognostic factor in esophageal squamous cell carcinoma, inhibits WIF-1 expression and activates Wnt pathway," Cancer Science, vol. 104, no. 12, pp. 1675-1682, 2013. 
[14] F. J. Chen, M. Sun, S. Q. Li et al., "Upregulation of the long non-coding RNA HOTAIR promotes esophageal squamous cell carcinoma metastasis and poor prognosis," Molecular Carcinogenesis, vol. 52, no. 11, pp. 908-915, 2013.

[15] C. H. Xu, Y. M. Guo, H. Y. Liu, G. B. Chen, Y. J. Yan, and T. Liu, "TUG1 confers cisplatin resistance in esophageal squamous cell carcinoma by epigenetically suppressing PDCD4 expression via EZH2," Cell \& Bioscience, vol. 8, no. 1, 2018.

[16] J. Ma, T. F. Li, X. W. Han, and H. F. Yuan, "Downregulated MEG3 contributes to tumour progression and poor prognosis in oesophagal squamous cell carcinoma by interacting with miR-4261, downregulating DKK2 and activating the Wnt/ $\beta$ catenin signalling," Artificial Cells, Nanomedicine, and Biotechnology, vol. 47, no. 1, pp. 1513-1523, 2019.

[17] H. Y. Deng, Y. C. Wang, P. Z. Ni, Y. D. Lin, and L. Q. Chen, "Long noncoding RNAs are novel potential prognostic biomarkers for esophageal squamous cell carcinoma: an overview," Journal of Thoracic Disease, vol. 8, no. 8, pp. E653E659, 2016.

[18] K. Zhang, Z. M. Shi, Y. N. Chang, Z. M. Hu, H. X. Qi, and W. Hong, "The ways of action of long non-coding RNAs in cytoplasm and nucleus," Gene, vol. 547, no. 1, pp. 1-9, 2014.

[19] L. Salmena, L. Poliseno, Y. Tay, L. Kats, and P. P. Pandolfi, “A ceRNA hypothesis: the Rosetta Stone of a hidden RNA language?," Cell, vol. 146, no. 3, pp. 353-358, 2011.

[20] M. D. Robinson, D. J. McCarthy, and G. K. Smyth, “edgeR: a bioconductor package for differential expression analysis of digital gene expression data," Bioinformatics, vol. 26, no. 1, pp. 139-140, 2009.

[21] C. H. Chou, N. W. Chang, S. Shrestha et al., "miRTarBase 2016: updates to the experimentally validated miRNA-target interactions database," Nucleic Acids Research, vol. 44, no. D1, pp. D239-D247, 2016.

[22] N. Wong and X. W. Wang, "miRDB: an online resource for microRNA target prediction and functional annotations," Nucleic Acids Research, vol. 43, no. D1, pp. D146-D152, 2015.

[23] B. Fromm, T. Billipp, L. E. Peck et al., "A uniform system for the annotation of vertebrate microRNA genes and the evolution of the human microRNAome," Annual Review of Genetics, vol. 49, no. 1, pp. 213-242, 2015.

[24] D. Szklarczyk, A. L. Gable, D. Lyon et al., "STRING v11: protein-protein association networks with increased coverage, supporting functional discovery in genome-wide experimental datasets," Nucleic Acids Research, vol. 47, no. D1, pp. D607D613, 2019.

[25] L. Yang, X. D. Zhao, and X. L. Tang, "Predicting diseaserelated proteins based on clique backbone in protein-protein interaction network," International Journal of Biological Sciences, vol. 10, no. 7, pp. 677-688, 2014.

[26] C. H. Chin, S. H. Chen, H. H. Wu, C. W. Ho, M. T. Ko, and C. Y. Lin, "cytoHubba: identifying hub objects and subnetworks from complex interactome," BMC Systems Biology, vol. 8, Supplement 4, p. S11, 2014.

[27] C. S. Huang, S. G. Liu, H. J. Wang, Z. C. Zhang, Q. Yang, and F. Gao, "LncRNA PVT1 overexpression is a poor prognostic biomarker and regulates migration and invasion in small cell lung cancer," American Journal of Translational Research, vol. 8, no. 11, pp. 5025-5034, 2016.

[28] H. Y. Jing, X. L. Qu, L. Y. Liu, and H. Xia, “A novel long noncoding RNA (lncRNA), LL22NC03-N64E9.1, promotes the proliferation of lung cancer cells and is a potential prognostic molecular biomarker for lung cancer," Medical Science Monitor Basic Research, vol. 24, pp. 4317-4323, 2018.

[29] E. E. Yoruker, M. Keskin, C. B. Kulle, S. Holdenrieder, and U. Gezer, "Diagnostic and prognostic value of circulating lncRNA H19 in gastric cancer," Biomedical Reports, vol. 9, no. 2, pp. 181-186, 2018.

[30] Y. Y. Zhang, R. Yang, J. C. Lian, and H. Y. Xu, "LncRNA Sox2ot overexpression serves as a poor prognostic biomarker in gastric cancer," American Journal of Translational Research, vol. 8, no. 11, pp. 5035-5043, 2016.

[31] Y. Jiao, Y. Q. Li, B. X. Jia et al., "The prognostic value of lncRNA SNHG4 and its potential mechanism in liver cancer," Bioscience Reports, vol. 40, no. 1, 2020.

[32] M. J. G. Milevskiy, F. al-Ejeh, J. M. Saunus et al., "Long-range regulators of the lncRNA HOTAIR enhance its prognostic potential in breast cancer," Human Molecular Genetics, vol. 25, no. 15, pp. 3269-3283, 2016.

[33] F. C. Beckedorff, M. S. Amaral, C. Deocesano-Pereira, and S. Verjovski-Almeida, "Long non-coding RNAs and their implications in cancer epigenetics," Bioscience Reports, vol. 33, no. 4, pp. 667-675, 2013.

[34] H. T. Liu, S. Liu, L. Liu, R. R. Ma, and P. Gao, "EGR1-mediated transcription of IncRNA-HNF1A-AS1 promotes cell cycle progression in gastric cancer," Cancer Research, vol. 78, no. 20, pp. 5877-5890, 2018.

[35] K. Zhang, J. Chen, H. Z. Song, and L. B. Chen, "SNHG16/miR140-5p axis promotes esophagus cancer cell proliferation, migration and EMT formation through regulating ZEB1," Oncotarget, vol. 9, no. 1, pp. 1028-1040, 2018.

[36] Y. Yan, S. Li, S. Wang et al., "Long noncoding RNA HAND2AS1 inhibits cancer cell proliferation, migration, and invasion in esophagus squamous cell carcinoma by regulating microRNA-21," Journal of Cellular Biochemistry, vol. 120, no. 6, pp. 9564-9571, 2018.

[37] A. Yamada, S. Fujii, H. Daiko, M. Nishimura, T. Chiba, and A. Ochiai, "Aberrant expression of EZH2 is associated with a poor outcome and P53 alteration in squamous cell carcinoma of the esophagus," International Journal of Oncology, vol. 38, no. 2, pp. 345-353, 2011.

[38] J. Lv, L. Guo, J. H. Wang et al., "Biomarker identification and trans-regulatory network analyses in esophageal adenocarcinoma and Barrett's esophagus," World Journal of Gastroenterology, vol. 25, no. 2, pp. 233-244, 2019.

[39] L. Lin, A. J. Bass, W. W. Lockwood et al., "Activation of GATA binding protein 6 (GATA6) sustains oncogenic lineagesurvival in esophageal adenocarcinoma," Proceedings of the National Academy of Sciences of the United States of America, vol. 109, no. 11, pp. 4251-4256, 2012.

[40] J. K. Mito, A. T. Agoston, P. Dal Cin, and A. Srivastava, "Prevalence and significance of HMGA2 expression in oesophageal adenocarcinoma," Histopathology, vol. 71, no. 6, pp. 909-917, 2017.

[41] Y. F. Wang, L. K. Sun, L. Wang et al., "Long non-coding RNA DSCR8 acts as a molecular sponge for miR-485-5p to activate Wnt/beta-catenin signal pathway in hepatocellular carcinoma," Cell Death \& Disease, vol. 9, no. 9, 2018.

[42] Q. You, Y. Yao, J. Wu, C. Cheng, Y. Li, and H. Yuan, "YY1induced lncRNA DSCR8 promotes the progression of ovarian cancer via miR-3192-5p/YY1 axis," Biomedicine \& Pharmacotherapy, vol. 129, article 110339, 2020. 
[43] E. A. Coe, J. Y. Tan, M. Shapiro et al., "The MITF-SOX10 regulated long non-coding RNA DIRC3 is a melanoma tumour suppressor," PLoS Genetics, vol. 15, no. 12, article e1008501, 2019.

[44] Y. Zhu, Y. Bian, Q. Zhang et al., "LINC00365 promotes colorectal cancer cell progression through the $\mathrm{Wnt} / \beta$-catenin signaling pathway," Journal of Cellular Biochemistry, vol. 121, no. 2, pp. 1260-1272, 2019. 